\title{
Assessment of the CTCF Binding Sites and Repeat-Positions Upstream the Human H19 Gene
}

Minou Bina

Department of Chemistry, Purdue University, West Lafayette IN 47907 USA

Key words: genomic imprinting, ICR, Imprinted Control Region, H19, IGF2, cohesin, CTCF, RAD21, SMC3

Correspondence: bina@purdue.edu

\section{ABSTRACT}

The human $\mathrm{H} 19$ and IGF2 genes share an Imprinting Control Region (ICR) that regulates gene expression in a parent-of-origin dependent manner. Understanding of the ICR sequence organization is critical to accurate localization of disease-associated abnormalities including Beckwith-Wiedemann and SilverRussell syndromes. Previous studies established that the ICR of the H19 - IGF2 imprinted domain included several repeated DNA segments. Using BLAST, BLAT, and Clustal Omega, I conducted detailed sequence comparisons to evaluate the annotation of the unique-repeats upstream of the $H 19$ transcription start site (TSS) and to investigate the extent of similarities among the various repeats. Initial analyses confirmed the existence of two DNA segments consisting of two types of repeats (A and $B$ ). However, I find that one of the repeats (B7) is unlikely to be a partial repeat. I provide the genomic positions of the various repeats in the build hg19 of the human genome. I also evaluated the previously predicted CTCF sites ( 1 to 7 ) in the context of the ENCODE data: including the positions of DNase I HS clusters and results of ChIP assays. My evaluations did not support the existence of CTCF site 5. Furthermore, the ENCODE data revealed a previously unknown chromatin boundary (consisting of CTCF, RAD21, and SMC3), in a CpG island (CpG27) between the A1 repeat and the H19 TSS. Furthermore, a sequence within this boundary corresponds to a newly discovered CTCF site (I named it CTCF site 8). My discovery of this chromatin boundary in CpG27 entails mechanistic implications.

\section{INTRODUCTION}

The Mammalian genomes include a group of genes that are imprinted to impart parent-oforigin-specific gene expression reviewed in (2-4). The human chromosomal band 11p15.5 encompasses an imprinted domain (H19 - IGF2) that is regulated by an ICR, at about $2 \mathrm{~kb}$ upstream of the H19 TSS. Nonmethylated ICR drives transcription from $\mathrm{H} 19$ gene in the maternal allele; ICR methylation leads to the expression of the IGF2 gene from the paternal allele (5). In humans, imprinting disorders cause developmental abnormalities including Beckwith-Wiedemann and Silver-Russell syndromes (6). in Silver-Russell syndrome up to $50 \%$ of methylation defects are in imprinted sequences on chromosome $11 \mathrm{p} 15$ (7). Abnormalities associated with Beckwith-Wiedemann syndrome include hypermethylation of the ICR that regulates the $H 19$ - IGF2 imprinted domain $(6,8)$.

In investigation of the H19 - IGF2 ICR, a previous study performed detailed BLAST analyses to define the upstream boundary of the imprinted DNA segment, and to determine whether Wilms tumors with loss of imprinting are biallelically CpG-methylated (1). This study identified several unique repeats. Since the location and annotation of these repeats are extensively used in studies of the human $H 19$ IGF2 ICR and developmental abnormalities, I wished to assess the accuracy of the repeats. I also wished to evaluate the ICR sequences implicated in binding the transcription factor $\operatorname{CTCF}(9,10)$. Specifically, it is well known that the ICR of the H19 - IGF2 interacts with the transcription factor CTCF to form 
chromatin boundaries $(5,11)$, and references therein. Usually, topological domains could arise through interactions among boundaries associated with CTCF and cohesin. Cohesin complexes are produced from SMC3, SMC1, RAD21, STAG1/SA1, and STAG2/SA2 (11-15). In cells, CTCF recruits a subset of cohesin subunits to affect chromatin architecture and gene expression $(11,13,15,16)$. CTCF largely determines the cohesin subunits that associated with transcriptionally active enhancers and promoters (15). Furthermore, cohesin and CTCF shape the organization of topological domains in non-redundant ways: while cohesin is important to shaping the domains, CTCF separates neighboring folding domains and keeps cohesin in place (11).

\section{RESULTS AND DISCUSSION}

\section{Organization of sequences upstream of the H19 TSS}

Previously, upstream sequences of the human $\mathrm{H} 19$ gene were found to contain two reiterated units (1 and 2) consisting of two 450-bp direct repeats ( $A 1$ and $A 2$ ) and several 400-bp repeats (B1 to $B 7$ ), two of which (B4 and B7) were reported to be incomplete (1). To validate the position and to determine the extent of similarities among the repeats, I obtained their annotations from the GenBank accession number AF125183, reported in reference (1). Initially, I mapped repeat-positions in the build hg19 of the human genome offered at the UCSC genome browser (Table 1). For assessment, I closely examined the relative repeat-positions with respect to genes, the ENCODE data (17), including the position of DNase I hypersensitive sites (HS) and results of chromatin immunoprecipitation assays (ChIPs) reported for CTCF, RAD21, and SMC3 $(17,18)$.

Table 1: genomic positions and length of A and B-repeats

\begin{tabular}{|l|l|l|}
\hline $\begin{array}{l}\text { Repeat } \\
\text { type }\end{array}$ & Genomic position in Build hg19 & $\sim$ Length \\
\hline A1 & chr11:2,020,617-2,021,067 & 451 bps \\
\hline B1 & chr11:2021068-2021474 & 407 bps \\
\hline B2 & chr11:2021475-2021880 & 406 bps \\
\hline B3 & chr11:2021881-2022287 & 407 bps \\
\hline B4p & chr11:2022346-2022481 & 136 bps \\
\hline A2 & chr11:2,022,868-2,023,312 & 445 bps \\
\hline B5 & chr11:2023313-2023714 & 402 bps \\
\hline B6 & chr11:2023715-2024120 & 406 bps \\
\hline B7p & chr11:2024163-2024414 & 252 bps \\
\hline B7L & chr11:2024121-2024516 & 396 bps \\
\hline
\end{tabular}

$\mathrm{B} 4 \mathrm{p}$ refers to $\mathrm{B} 4$ partial, $\mathrm{B} 7 \mathrm{p}$ to $\mathrm{B} 7$ partial, $\mathrm{B} 7 \mathrm{~L}$ to a nearly complete $\mathrm{B} 7$ repeat. 
To evaluate the reported repeats (1), I performed extensive sequence similarity searches using BLAST (19), BLAT (20), and Clustal Omega (21). Initially, I analyzed overlapping genomic DNA segments derived from the region between the $\mathrm{H} 19$ and IGF2 genes. I found that overall results obtained from BLAST were relatively accurate but its pitfalls included outputs that were very labor intensive to analyze. Furthermore, it seemed that BLAST omitted short sections of sequence similarities that could be important. The pitfall of BLAT was that its outputs were bizarre since the program was not designed for analyzing unique repeated DNA. While Clustal $O$ was relatively convenient to use, the program could not handle relatively long sequences. Nonetheless, outputs of Clustal O provided a robust way for closely examining the extent of sequence similarities between two or several aligned sequences.

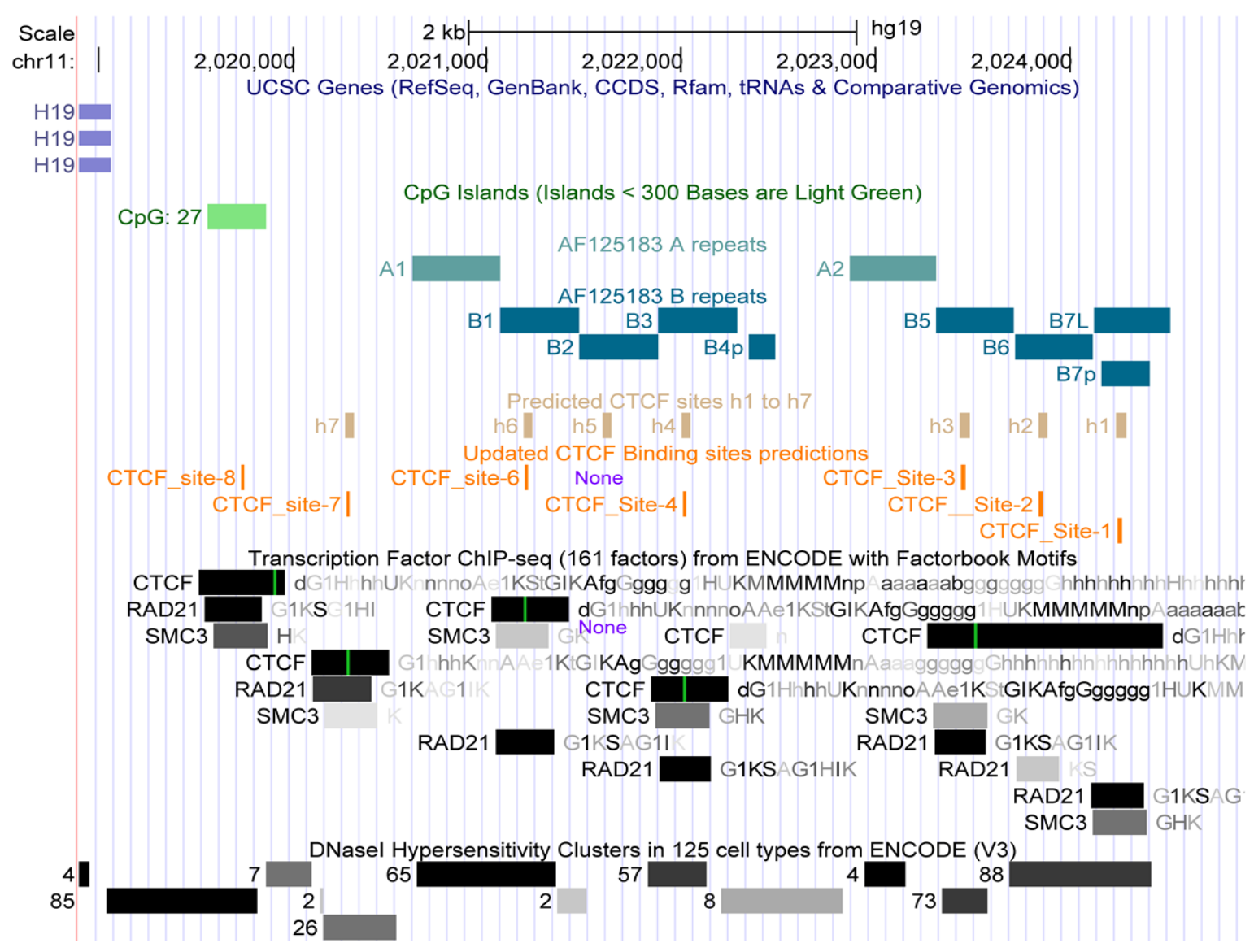

Fig. 1. A snapshot from the UCSC genome browser displaying the positions of $A$ and $B$ repeats with respect to H19 TSS. This figure shows the positions of h1-h7 reported in reference (5), my predicted CTCF sites in the context of results of the ENCODE ChIPs (reported for CTCF, RAD21, and SMC3), and DNase I hypersensitive clusters. Note that results of ChIPs did not find any association of CTCF with h5 (previously reported as CTCF site 5). Also note the position of a previously unknown chromatin boundary (consisting of CTCF, RAD21, and SMC3) in results of the ENCODE ChIP assays reported for the build hg19 of the human genome (17). This boundary includes a predicted CTCF site (designated site 8). 
With Clustal O, initially I assessed the relative repeat positions, by comparing the nucleotide sequences of two DNA sections that I downloaded from the UCSC genome browser: one section encompassed A1, B1, B2, B3, and B4; the other A2, B5, B6, and B7 (Appendix A). For evaluation, I obtained repeat-annotations from reference (1), listed in GenBank accession number AF125183. In agreement with that report, my analyses identified two classes of unique repeats ( $A$ and $B$ ), positioned with respect to the TSS of the human $\mathrm{H} 19$ gene (Fig. 1). These two repeat classes are within a duplicated DNA segment: repeat unit 1 and repeat unit 2, reference (1). Unit 1 is proximal to the $H 19$ transcription site and includes A1, B1, B2, and B3; Unit 2 is upstream of the first unit and includes A2, B5, B6, and B7; between the two units, there is a partial repeat (B4), designated B4p in Fig. 1. In initial pairwise alignments, I noted that several of the previous assignments were accurate, vis-à-vis start and end positions of the following pair of sequences: $A 1$ and A2; B1 and B5; B2 and B6 (Appendix A). However, even though from results of BLAST, one could deduce that B7 was a partial repeat (1), Clustal $O$ alignments of $B 3$ and $B 7$ partial suggested that B7 corresponded to a somewhat full repeat (see the $2^{\text {nd }}$ and the $3^{\text {rd }}$ pages in Appendix A). Specifically: the start position of B3 in repeat unit 1 shares a stretch of sequence similarity with the corresponding region in repeat unit 2; likewise, the end position of B3 in repeat unit 1 shares a stretch of sequence similarity with the corresponding region in repeat unit 2 .

To further evaluate the accuracy of B7 corresponding to a partial repeat, I performed Dotpath analysis. In this and related methods, a program creates a two-dimensional matrix for a pair of sequences, placed along the $X$ and $Y$ axes (Fig. 2). Dotpath simultaneously scans both sequences in a specified window. In the program's output, sequences that match appear as dots (22). Homologous sequences produce a diagonal line in the center of the matrix; mismatches, including insertions and deletions, cause disruptions in the diagonal. Overall, results of Dotpath analysis of B3 against the long version of B7 (B7L) gave a nearly diagonal line; a few mismatches are detectable in the vicinity of the center of the diagonal (Fig. 2); offset from the diagonal is due to a small deletion within B7L, producing a sequence somewhat shorter than the one obtained for B3 (Appendix A and Table 1).

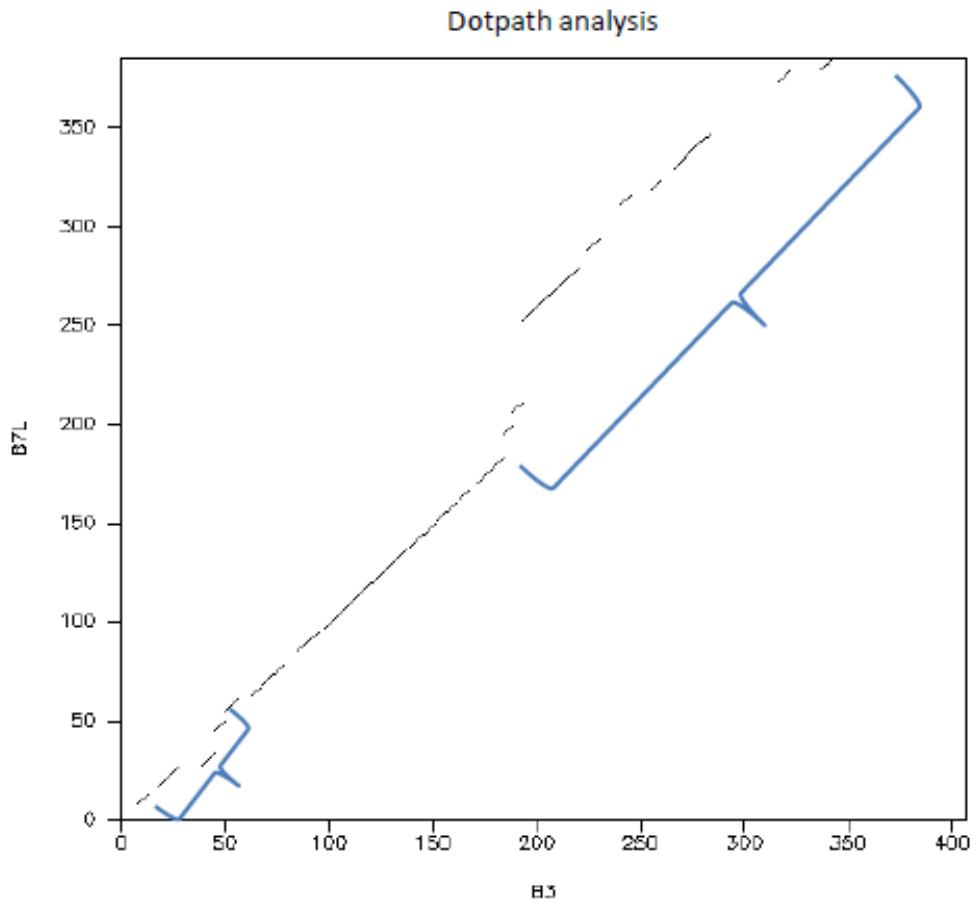

Braces mark the B7 flanking sequences, producing B7L
Fig. 2. Result of Dotpath analysis indicates that B7 is probably not a partial sequence. Two braces mark the position of sequence similarities obtained from analyses done using Clustal $O$ and Dotpath. These similarities were not detected in results of studies detailed in a previous report (1). 


\section{Multiple sequence-alignment analyses}

Comparative sequence-alignments of B repeats could help with examining regulation of the $H 19$ ICR-mediated transcription and pinpointing the molecular basis of reported genetic abnormities, including Beckwith-Wiedemann syndrome. Towards this goal, I performed several sets of CLUSTAL O alignments of files created to include various combinations of sequences corresponding to $B$ repeats. An initial set contained B1, B2, B3, B4, B5, B6, B7p, and B7L (the longer form of B7). This set was done to determine the missing part in $B 4$ and to display the sequence of $B 7 L$ with respect to its shorter form obtained in a previous annotation (1). I found that the section missing from B4 is relatively long and corresponds to a $3^{\prime}$ end deletion, producing B4p (Appendix B). Next I examined a set consisting of B1, $B 2, B 3, B 5, B 6$, and B7L while omitting B4p and B7p (Appendix C). This analysis was done to determine the extent of similarity of B7L to B1, B2, B3, B5, and B6. At first glance, it appeared that the $5^{\prime}$ end of B7L shared a limited similarity to the corresponding region in other repeats. However, upon close inspections, I noted several identical nucleotides in this section of $B 7 L$ to the $5^{\prime}$ end of other repeats (shown in bold in Appendix C). Furthermore, in a set that does include B7L, it is evident that the $5^{\prime}$ end sequences of other repeats vary greatly and thus share a limited similarity (Appendix D). Also, CLUSTAL $\mathrm{O}$ alignments located the position of a small internal deletion in B7L, detected in results of Dotpath analyses (Fig. 2 and Appendix C); likewise, CLUSTAL $O$ alignments found a 4 base-pair deletion in B5, as well as 1-base insertions/deletions at various repeat-positions (Appendix C). Thus, overall, results of both Dotpath and CLUSTAL $O$ indicate that B7L is nearly a complete repeat, even though it contains a relatively short internal deletion. This deletion explains why B7L is somewhat shorter than B1, B2, B3, B5, and B6 (Table 1).

\section{Evaluation of predicted CTCF binding sites}

The transcription factor CTCF is an 11 zinc-finger DNA-binding protein that exerts maternal effects on transcription during mammalian oocyte growth, and plays important roles in meiotic maturation and early embryonic development (23). Association of CTCF with a subset of cohesin subunits may contribute to the topological domain architecture and to the formation of regions that exhibit unusually high levels of local chromatin interactions (24). A study predicted 7 CTCF binding regions (h1 to h7), within a segment upstream of the human $\mathrm{H} 19$ gene (10), summarized in (Fig. 3). In the H19/IGF2 imprinted domain, CTCF binding sites are thought to contribute to formation of chromatin boundaries in the maternal allele $(5,9,11)$. Notably, many publications have relied on the predicted CTCF sites in studies of both Beckwith-Wiedemann and Silver-Russell syndromes. Because of the importance of CTCF to the regulation of the H19 - IGF2 imprinted domain, I wished to evaluate the genomic positions of $\mathrm{h} 1$ to $\mathrm{h} 7$, in the context of the ENCODE data: including the position of DNase I HS clusters (HS-CS) in chromatin and results of ChIPs obtained for CTCF and two of the cohesin subunits (Rad21 and SMC3). ENCODE (V3) reported DNase I HS-Cs for 125 human cell types (25). The chromatin section that encompasses the H19 TSS, includes several prominent DNase I HS-Cs: i.e. HS-C 85, 65, 57, 4, 73,88 , and 10 (Fig. 1); a numerical value indicates the number of cell-lines containing a HS-C mapping to a specific genomic location. HS-C 85 encompasses the $\mathrm{H} 19$ TSS and extends to include part of a CpG island (CpG27); HS-C 65 overlaps with A1 and B1; HS-C 57 overlaps with B3; HS-C 4 is within A2; HS-C 73 is within B5; HS-C 88 overlaps with B7L and encompasses B6 (Fig. 1). HS-C 10 is further upstream (not shown). Notably, I did not find any HS-C associated with B2, which includes h5, corresponding to the predicted CTCF binding site 5 (Fig. 1). 
The inconsistency noted above led me to closely inspect the genomic positions of h1 to h7 with respect to results of the ENCODE ChIPs, focusing on data reported for CTCF and two cohesin subunits. I also analyzed sequences upstream of the $\mathrm{H} 19$ gene, for potential CTCF binding sites, using a webserver developed and maintained by Yan Cui's Lab, at the University of Tennessee Health Science Center (26): http://insulatordb.uthsc.edu/. Fig. 1 shows CTCF binding sites predicted by that server; they are displayed on a track labeled 'updated CTCF Binding sites predictions'. With respect to the ENCODE data, CTCF binding sites 1 to 3 map to a relatively broad CTCF-associated region (Fig. 1). ChIP data further revealed that both site 1 and 3 were associated with the cohesin subunit RAD21; site 3 might also be associated with SMC3 (Fig. 1). Sites 4, 6, and 7 are primarily associated with CTCF and RAD21 (Figs. 1 and 3). Thus, among the previously predicted CTCF sites only h5 (in repeat B2) did not map to a CTCF-bound region, according to results of the ENCODE ChIP assays (Fig. 1). To obtain clues into the reason for a lack of CTCF association with $\mathrm{h} 5$, I inspected the sequences within the B1 to B7 repeats (Appendix C), summarized in Fig. 3. A close-up view of Clustal O alignment revealed nearly $100 \%$ identity in the repeat-sequences encompassing predicted CTCF binding sites. Within that range I noted a $\mathrm{G}$ to $\mathrm{A}$ mutation in repeat $\mathrm{B} 2$, the previously predicted CTCF site 5 in h5; another mutation in B2 (T to $\underline{\mathrm{C}}$ ) might also contribute to or responsible for the lack of CTCF association with B2. This interpretation agrees with results of the ENCODE ChIP assays (Figs 1 and 3). Noteworthy could be clinically associated SNPs including rs431825167 and rs483353061 (both in B1) and rs431825169 (in B2).

Fig.3. CLUSTAL O alignment of updated, predicted CTCF binding sites in the human H19 ICR Sequences are shown in the direction found in the chromosomal DNA

Updated, predicted CTCF binding sites CTCF ChIP

\begin{tabular}{|c|c|c|c|}
\hline B1 & TGAGCCTGCACTGCCGCCGCGCGGCCACTTCCGA & 6 & Yes \\
\hline B2 & TGAGCCTGCACTGCCGCCGCGCAGGCACㅡTCCGA & - & No \\
\hline B3 & TGAGCCTGCACTGCCGCCACGCGGCCACTTCCGA & 4 & Yes \\
\hline B5 & TGAGCCTGCACTGCCGCCGCGCGGCCACTTTTGA & 3 & Yes \\
\hline B6 & GGAGCCTGCACTGCCGCCGCGCGGCCACTTCCGA & 2 & Yes \\
\hline B7 & TGAGCCTGCACTGCCGCCGCGCGGCCACTTCTGA & 1 & Yes \\
\hline
\end{tabular}

According to ENCODE ChIPs (11), B2 was not associated with CTCF in the examined cell lines (Fig. 1). It appears that a $\mathbf{G}$ to $\underline{\mathbf{A}}$ mutation (in B2) has abolished or reduced the affinity of CTCF for the DNA. However, another $\mathbf{G}$ to $\underline{\mathbf{A}}$ mutation in B3 did not obliterate the association of CTCF with B3. It remains possible that another mutation in $\mathrm{B} 2$ ( $\mathrm{T}$ to $\underline{\mathrm{C}}$ ) might also contribute to the lack of CTCF association with $\mathrm{B} 2$. 
Notably, results of the ENCODE ChIPs also revealed that a CpG island, upstream of the $H 19$ gene (CpG27), includes a previously unknown chromatin boundary consisting of CTCF, RAD21, and SMC3 (Fig. 1). Within that region, the Yan Cui's server (26) predicted a CTCF binding site with a DNase I HS cluster: HS-C 85 (Fig. 1). I named the site (CTCF site 8). Clearly, a lack of CTCF association with h5 and the discovery of a chromatin boundary within CpG27, entail mechanistic implications regarding how formation of loops and topological domains would regulate allele-specific gene expression from the $H 19$ - IGF2 imprinted domain.

Appendices $A$ to $D$ are shown after the references.

\section{REFERENCES}

1. Frevel, M.A., Sowerby, S.J., Petersen, G.B. and Reeve, A.E. (1999) Methylation sequencing analysis refines the region of $\mathrm{H} 19$ epimutation in Wilms tumor. J Biol Chem, 274, 29331-29340.

2. Plasschaert, R.N. and Bartolomei, M.S. (2014) Genomic imprinting in development, growth, behavior and stem cells. Development, 141, 1805-1813.

3. Wan, L.B. and Bartolomei, M.S. (2008) Regulation of imprinting in clusters: noncoding RNAs versus insulators. Advances in genetics, 61, 207-223.

4. Cleaton, M.A., Edwards, C.A. and Ferguson-Smith, A.C. (2014) Phenotypic outcomes of imprinted gene models in mice: elucidation of pre- and postnatal functions of imprinted genes. Annual review of genomics and human genetics, 15, 93-126.

5. Lewis, A. and Murrell, A. (2004) Genomic imprinting: CTCF protects the boundaries. Curr Biol, 14, R284-286.

6. Soejima, H. and Higashimoto, K. (2013) Epigenetic and genetic alterations of the imprinting disorder Beckwith-Wiedemann syndrome and related disorders. Journal of human genetics, 58, 402-409.

7. Abu-Amero, S., Monk, D., Frost, J., Preece, M., Stanier, P. and Moore, G.E. (2008) The genetic aetiology of Silver-Russell syndrome. Journal of medical genetics, 45, 193-199.

8. Engel, J.R., Smallwood, A., Harper, A., Higgins, M.J., Oshimura, M., Reik, W., Schofield, P.N. and Maher, E.R. (2000) Epigenotype-phenotype correlations in Beckwith-Wiedemann syndrome. Journal of medical genetics, 37, 921-926.

9. Hark, A.T., Schoenherr, C.J., Katz, D.J., Ingram, R.S., Levorse, J.M. and Tilghman, S.M. (2000) CTCF mediates methylation-sensitive enhancer-blocking activity at the H19/Igf2 locus. Nature, 405, 486-489.

10. Bell, A.C. and Felsenfeld, G. (2000) Methylation of a CTCF-dependent boundary controls imprinted expression of the Igf2 gene. Nature, 405, 482-485.

11. Zuin, J., Dixon, J.R., van der Reijden, M.I., Ye, Z., Kolovos, P., Brouwer, R.W., van de Corput, M.P., van de Werken, H.J., Knoch, T.A., van, I.W.F. et al. (2014) Cohesin and CTCF differentially affect chromatin architecture and gene expression in human cells. Proc Natl Acad Sci U S A, 111, 9961001.

12. Dixon, J.R., Selvaraj, S., Yue, F., Kim, A., Li, Y., Shen, Y., Hu, M., Liu, J.S. and Ren, B. (2012) Topological domains in mammalian genomes identified by analysis of chromatin interactions. Nature, 485, 376-380.

13. Rubio, E.D., Reiss, D.J., Welcsh, P.L., Disteche, C.M., Filippova, G.N., Baliga, N.S., Aebersold, R., Ranish, J.A. and Krumm, A. (2008) CTCF physically links cohesin to chromatin. Proc Natl Acad Sci U S A, 105, 8309-8314. 
14. Nativio, R., Wendt, K.S., Ito, Y., Huddleston, J.E., Uribe-Lewis, S., Woodfine, K., Krueger, C., Reik, W., Peters, J.M. and Murrell, A. (2009) Cohesin is required for higher-order chromatin conformation at the imprinted IGF2-H19 locus. PLoS Genet, 5, e1000739.

15. Parelho, V., Hadjur, S., Spivakov, M., Leleu, M., Sauer, S., Gregson, H.C., Jarmuz, A., Canzonetta, C., Webster, Z., Nesterova, T. et al. (2008) Cohesins functionally associate with CTCF on mammalian chromosome arms. Cell, 132, 422-433.

16. Wendt, K.S., Yoshida, K., Itoh, T., Bando, M., Koch, B., Schirghuber, E., Tsutsumi, S., Nagae, G., Ishihara, K., Mishiro, T. et al. (2008) Cohesin mediates transcriptional insulation by CCCTCbinding factor. Nature, 451, 796-801.

17. ENCODE. (2011) A user's guide to the encyclopedia of DNA elements (ENCODE). PLoS Biol, 9, e1001046.

18. Wang, J., Zhuang, J., Iyer, S., Lin, X.Y., Greven, M.C., Kim, B.H., Moore, J., Pierce, B.G., Dong, X., Virgil, D. et al. (2013) Factorbook.org: a Wiki-based database for transcription factor-binding data generated by the ENCODE consortium. Nucleic Acids Res, 41, D171-176.

19. Altschul, S.F., Gish, W., Miller, W., Myers, E.W. and Lipman, D.J. (1990) Basic local alignment search tool. J Mol Biol, 215, 403-410.

20. Kent, W.J. (2002) BLAT--the BLAST-like alignment tool. Genome Res, 12, 656-664.

21. Sievers, F. and Higgins, D.G. (2014) Clustal Omega, accurate alignment of very large numbers of sequences. Methods Mol Biol, 1079, 105-116.

22. Gibbs, A.J. and McIntyre, G.A. (1970) The diagram, a method for comparing sequences. Its use with amino acid and nucleotide sequences. European journal of biochemistry, 16, 1-11.

23. Wan, L.B., Pan, H., Hannenhalli, S., Cheng, Y., Ma, J., Fedoriw, A., Lobanenkov, V., Latham, K.E., Schultz, R.M. and Bartolomei, M.S. (2008) Maternal depletion of CTCF reveals multiple functions during oocyte and preimplantation embryo development. Development, 135, 2729-2738.

24. Schmitt, A.D., Hu, M., Jung, I., Xu, Z., Qiu, Y., Tan, C.L., Li, Y., Lin, S., Lin, Y., Barr, C.L. et al. (2016) A Compendium of Chromatin Contact Maps Reveals Spatially Active Regions in the Human Genome. Cell Rep, 17, 2042-2059.

25. ENCODE. (2012) An integrated encyclopedia of DNA elements in the human genome. Nature, 489, 57-74.

26. Ziebarth, J.D., Bhattacharya, A. and Cui, Y. (2013) CTCFBSDB 2.0: a database for CTCF-binding sites and genome organization. Nucleic Acids Res, 41, D188-194.

Appendices $A$ to $D$ are listed in subsequent pages. 
bioRxiv preprint doi: https://doi.org/10.1101/250407; this version posted January 19,2018 . The copyright holder for this preprint (which was not certified by peer review) is the author/funder, who has granted bioRxiv a license to display the preprint in perpetuity. It is made available under aCC-BY-ND 4.0 International license.

Appendix A: output of CLUSTAL 0 (http://Www.ebi.ac.uk/Tools/msa/clustalo/), analyzing 2 large genomic DNA sections that included the reported A and B repeats. This appendix includes pairwise comparison of the reported repeats units 1 and 2, the reported positions described in the GenBank accession number AF125183, and my comments (this output consists of 3 pages). Note the extend of sequence similarities and the position of insertions and deletions (INDELS)

\section{$\downarrow$ Start of A1 (accurate)}

Before_A1_to-after_B3 Before_A2_to-after_B7

Before_A1_to-after_B3 Before_A2_to-after_B7

Before_A1_to-after_B3 Before_A2_to-after_B7

Before_A1_to-after_B3 Before_A2_to-after_B7

Before_A1_to-after_B3 Before_A2_to-after_B7

Before_A1_to-after_B3 Before_A2_to-after_B7

Before_A1_to-after_B3 Before_A2_to-after_B7

Before_A1_to-after_B3 Before_A2_to-after_B7

Before_A1_to-after_B3 Before_A2_to-after_B7

Before_A1_to-after_B3 Before_A2_to-after_B7

Before_A1_to-after_B3 Before_A2_to-after_B7

Before_A1_to-after_B3 Before_A2_to-after_B7

Before_A1_to-after_B3 Before_A2_to-after_B7
- CATCCTAACCCCATCAGGATCCCCACCCCGTACCGAGTCCACAAGCCGCAGGGTGTCTG CAGTGCACAAACACCATTGATCCCCATCCTTTATCGATTCCACAAACCGCGGGATGTCTG $* * * * \quad * * * * * * * * * \quad * * * * * * * * * * * * * * * * * * * * * * * *$ $\uparrow$ Start of A2 (accurate)

CTGCACCCACGATAATGGATTATCTCCAGCAACACAAGGATCCTGGACCAGAGAATAAAG CTGCACCCACATTAGCAGATTATCTCAGCCAACACAAGGATCCTAGACCCCAGAATAAAG $* * * * * * * * * * \quad * * \quad * * * * * * * * * \quad * * * * * * * * * * * * * * * * * * * \quad * * * * * * * * *$

CAGCAGTGGCGCTCCCAGCTCTTTAGCATCTTAAGCTCCTGAATTAGCACCTCAAACCTG CAGCAGTGGCACTCCCAGCTCTTTAGCATCTCAAGCTCCTAAATTAGCATCTCAAACCTG $* * * * * * * * * * * * * * * * * * * * * * * * * * * * * * * * * * * * * * * * * * * * * * * * * * * * * * * *$

CATTGAATGAGAATGTTAATGTCTGGCCACTTAGGGCTGAGGAGATCAGAGTGTTCACTG CACTGAATGGGAATGTTAATGTCTGGCCACTTAAGATTGACAAGCTCAGAGTGTTCACTG $* * * * * * * * * * * * * * * * * * * * * * * * * * * * * * * * * * * \quad * * * * * * * * * * * * * * * * *$

GGGAGGCAATTGTCAGTTCAGTAAAAGGCTGGGGATTTGGGGGCTGTCCTTAGACGGAGT GGGAGGCAATTGTCAGTTCAGAAAAAGGCTCGGGATTTGGGGGCTGTCCTTAGTC - - - - $* * * * * * * * * * * * * * * * * * * * * * * * * * * * * \quad * * * * * * * * * * * * * * * * * * * * * * *$

CGGAGCTGTGCTCTGGGATAGATGTGGAAAATGTAAGATTTTGGTGGAACACACTGTGAT - GGATCTGTGCTCTGGGATGGATGTGGAAAGTATAAGCTTTTGGCGGAATTTGCTGTGCT $* * * * * * * * * * * * * * * * * * * * * * * * * * * * * * * * * * * * * * * * * * \quad * * * * * *$

CATCACATAAGTAGGCGTGACTTGAGTCCCAGGCCATGACACTGAAGCCCTCGGAGTGTG CATCACGCGGGTAGGCGTGACTTGAGTCCCAGGCCATGACACTGAAGCCCTCAGAGTGTG $* * * * * * \quad * * * * * * * * * * * * * * * * * * * * * * * * * * * * * * * * * * * * * * * * * * * * * * * * *$

(Accurate) End of A1 $\downarrow$ Start of B1

ACCCGGGGCCACGGGGCTGTGGATAATGCCCGACCTGAAGATCTGGTGCGGCTCCCATGA ACCTGGGGCCACGCGGCCGTGGATATGGCCCGATACGAAGACGTGGTGTGGCTCCCATGA $* * * * * * * * * * * * * * * * * * * * * \quad * * * * * * \quad * * * * * \quad * * * * * * * * * * * * * * * *$

(Accurate) End of A2 $\uparrow$ Start of B5

GTGTCCTATTCCCAGATGACCCCCGTGAACCCTGCGACGCGTGGCTTGGGTGACCCGGGA GCGTCCTATTCCCAGAAGACCTCCGAGAACCCTGCGGCACCTGGCTTGGGTGACCCAGGA

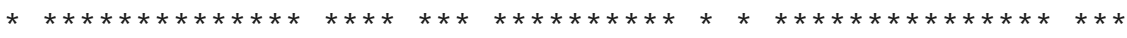

CGTTTCCACGGGCGAACCCCAGTTGGGGCGGGCTCGGGCTGTGATGTGTGAGCCTGCACT CGTTTCCGCGGGCGAACCCCATCCAGGGAGGGCTTGGGCTGTGATGTGTGAGCCTGCACT $* * * * * * * * * * * * * * * * * * * * \quad * * * \quad * * * * * * * * * * * * * * * * * * * * * * * * * * * * * *$

GCCGCCGCGCGGCCACTTCCGATTCCACAACTACAACCAATTCCGTGCCATCCAGGCGGT GCCGCCGCGCGGCCACTTTTGAGTCCACACTTACAACCGATTCTGTGCCATCCGGGTGGT $* * * * * * * * * * * * * * * * * * \quad * * * * * * * * \quad * * * * * * * * * * * * * * * * * * * * * * * * *$

GAGACCGAAGGAGAAGCCTCCAGAAATACCCATGTGCTATGCAAGAGCCCCCGGGCTCTG GAGACAGAAGGGGAAGCGTCCACAAATACCCATGTGCTACGCAAGAGCCCCCAGTTTCTG $* * * * * * * * * * * * * * * * * * * * * * * * * * * * * * * * * * * * * * * * * * * * * * * * * * * *$

TGCCTGCCAGAGACCCTGCGAGAAGGGTTTCACACTAGGGCCGAGATCCCCATCATCCAT TGCCTGCCAGAGACCCTGTGGGAGGGGCCTCACGCTGAGGCCGAGATCCCCATCATCCAA $* * * * * * * * * * * * * * * * * * * * * * *$ *****t* $* * * * * * * * * * * * * * * * * * * * *$ 
bioRxiv preprint doi: https://doi.org/10.1101/250407; this version posted January 19,2018 . The copyright holder for this preprint (which was not certified by peer review) is the author/funder, who has granted bioRxiv a license to display the preprint in perpetuity. It is made available under aCC-BY-ND 4.0 International license.

Before_A1_to-after_B3 Before_A2_to-after_B7

Before_A1_to-after_B3 Before_A2_to-after_B7

Before_A1_to-after_B3 Before_A2_to-after_B7

Before_A1_to-after_B3 Before_A2_to-after_B7

Before_A1_to-after_B3 Before_A2_to-after_B7

Before_A1_to-after_B3 Before_A2_to-after_B7

Before_A1_to-after_B3 Before_A2_to-after_B7

Before_A1_to-after_B3 Before_A2_to-after_B7

Before_A1_to-after_B3 Before_A2_to-after_B7

Before_A1_to-after_B3 Before_A2_to-after_B7

Before_A1_to-after_B3 Before_A2_to-after_B7

Before_A1_to-after_B3 Before_A2_to-after_B7

Before_A1_to-after_B3 Before_A2_to-after_B7

Before_A1_to-after_B3 Before_A2_to-after_B7
GGAACTGGGACACCTCATTGTTCCCCTAGTATCTCCTCCCATCTCCCCAACCTTCAACAG AGAATGGGGACACCTCATTGTCACCAAAGTATCTC - - - - ATCTCCCCAACCCTCAATAG $* * * \quad * * * * * * * * * * * * * * * \quad * * \quad * * * * * * * * \quad * * * * * * * * * * * * * * * * * *$

End of B1 $\downarrow$ Start of B2 (accurate) TGCACCCTGGGGTGAATCAGACACGTAGCCCGATATGGCTCCCATGAGTGTCCTATACCT TGCACCCTGGGGTGAATCAGACACGTAGCCCAATGTGGCTCCCATGAGTGTTCTATCCCT End of B5 Start of B6 (accurate)

CACGACCCCTGTGAACCCTGTGGCGCCCGGCTTGGATGACCTGGGATGTTTCCACGGGCG CACTACCCCCGTGAACCTTGCGGCACCTAGCTTGCGTGACCCGGGACGTTTCCGCGGGTG

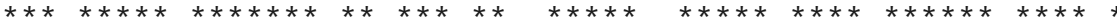

AACCCCAGCTGGGGCGGGCTCAGGCTGTGATGTGTGAGCCTGCACTGCCGCCGCGCAGCC AACCTCAGCTGGGGCGGGCTTGAGCTGTGATGTGGGAGCCTGCACTGCCGCCGCGCGGCC

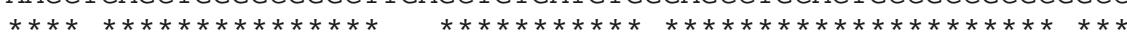

ACCTCCGATTCCACAACTACAACCAATTCTGTGCCATCCGGGCGGTGAGACTGAAGGGGA ACTTCCGATTCCACAACTACAACCGATTCTGCACCATCGGGGCGGTGAGACCGAAGGGGA $* * * * * * * * * * * * * * * * * * * * * * * * * * * * * * * * * * * * * * * * * * * * * * * * * * * * * *$

AGCCTCCAGAAATACACATGTGCTATGCAAGAGCCCCCGGGCTCTGTGCCTGCCAGAGAC AGCCTCCAGAAATACACATGTGCTATGCAAGAGCCCCCAGGCTCTGTGCCTGCCAGAGAC

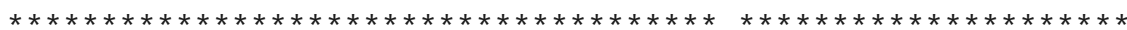

CCTGCAAGAAGGATCTCACCCTGAGgCCAAGATCCCCATCATCCAAAGAATGGGGACACT CCTGTAGGAGGGGTCTCACGCTTAGGCCGAGATCCCTATCATCCAAGGAACTGGGACACC

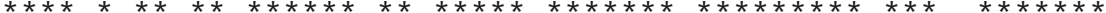

TCATTGTCCCCCAAGTATCTCСTCTCATCTCCCCAACCCTCAATAGTGCACCCTGGGGTG TCATTGTCCCCCAAGCATCTCCTCCAATCTCCCCAACCTTCAACAATGCACCCTGGGGTG $* * * * * * * * * * * * * * * * * * * * * * * \quad * * * * * * * * * * * * * * * * * * * * * * * * * * * * * * *$

End of B2 $\downarrow$ start of B3 (accurate) AATCAGACACATAGCCCGATGTGGCTCCCATGAATGTCCTATCCCTGATGACCCCCGTGA AATCAAACACATAGCCCGATGTTAGTCGCATGAGTGTCTATCTCTGACAACCCTCGGGAA $* * * * * * * * * * * * * * * * * * * * * * * * * * * * * * *$ * $* * * *$ End of B6 $\uparrow$ Likely start of B7L (see result of dotpath)

ACCCTGCGGCGCCTGGCTTGCGGGACCCGGGACGTTTCCGCGGGCGAACCCCAGCTGGGG CCAATGCGGCACCTGGCCTGGGAGACCTGGGACGTTTCTGTGGGTGAACCCCA-GCCAGG $* \quad * * * * * * * * * * * * * * * * * * * * * * * * * * * * * * * * * * * * * * * * * \quad * *$ $\uparrow$ Reported start of B7p (probably inaccurate)

CGGGCTCGGGCTGTGATGTGTGAGCCTGCACTGCCGCCACGCGGCCACTTCCGATTCCAC CGTGCTCGGGCTGTGATGTGTGAGCCTGCACTGCCGCCGCGCGGCCACTTCTGATTCCAC

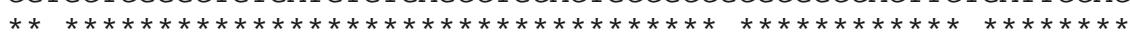

AGCTACAACCAATTCTGCACCATCCGGGTGGTGAGACTGAAGGGGAAGCCTCCAGAAATA ACGTACAGCCGATTCTGCGCCATCAGGGCAGTGAGACGGGCCTCCCTTGGTGAGGTCAGC

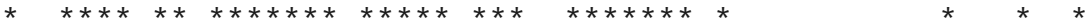

TTCATGTGCTATGCAAGAGCCCCTGGGCTCTTGAGCCTGCCAGAGACCCTGTGGGAGGGG AAGATGGGCCGCCCTCGGTGAGGCTGGCATCCAAGACTGAATGGGAAGCCTCCAGAAATA $* * * * * * * * * * * * * * * *$

TCTCACGCTGAGGCCGAGATCCCCATCATCCAAAGAATGGGGACACTTCACTGTCCCCCA CCTGTGCGCTGTGCTTGGCAGGCTCCCA - - - - CGATCCCTGTTCC - - - - - - - - - TGC

Reported end of B7p

(deletion in B7L) 
bioRxiv preprint doi: https://doi.org/10.1101/250407; this version posted January 19, 2018. The copyright holder for this preprint (which was not certified by peer review) is the author/funder, who has granted bioRxiv a license to display the preprint in perpetuity. It is made available under aCC-BY-ND 4.0 International license.

Before A1 to-after B3

Before_A2_to-after_B7

Before_A1_to-after_B3

Before_A2_to-after_B7

Before_A1_to-after_B3

Before_A2_to-after_B7

Before_A1_to-after_B3

Before_A2_to-after_B7

Before_A1_to-after_B3

Before_A2_to-after_B7

Before_A1_to-after_B3

Before_A2_to-after_B7
AGTATCTCCTCTCATCTCCCCAACCTTCAACAGTGCACCCTGGGGTGAATCAGGCACGTA TGGA - - - - - GGCCCTGTTGGGAGGGTCTCCCTCTGGACCTTGGGCTCAAGTTCTTTGGGG

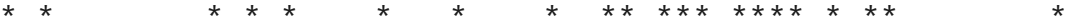

Extension in B7L

End of B3

GCCCGATG - - - - - - - - TGGCTCGTACAAGGGTCCTACCCCCGATGACCCTCGCGAACC TCCAAGTCATGACCACTGCAGAACAGAGATTTCTCTTTGCCTCCAAGTATCCACTGTCCT **

Likely end of B7L

AATGCGGTGCCTGGCCTGTTGACCCGGGATGTTTCTGCAGGCAAACCACAGGTAGCCGGG

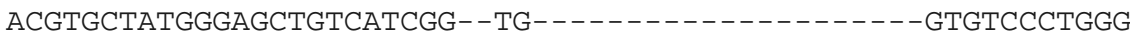
$* x_{* *}+t_{*} * * *$

CTCGGGCTATGACGCCTGAGCCTGCCCTGCTACCACGTGGCCACCTGTGTTTCCATACCT ATTAGG - _ - $\ldots$ * $\quad *$

TCAACTGATTCCGTGGCATCTGGGCAGCGAGACTCCAGGAACACTGTGCGCTCTGCTTGG

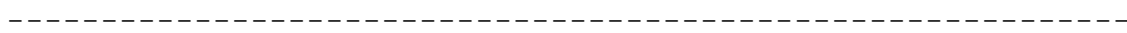

CAGGCTCCCAGGATCCCTGTTCCTGCCAAAG

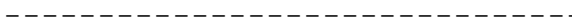


bioRxiv preprint doi: https://doi.org/10.1101/250407; this version posted January 19,2018 . The copyright holder for this preprint (which was not certified by peer review) is the author/funder, who has granted bioRxiv a license to display the preprint in perpetuity. It is made available under

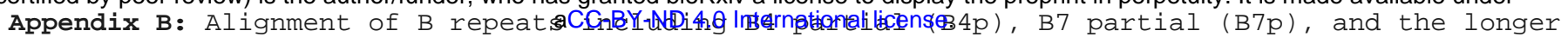
form of $B 7(B 7 L)$. Shown in blue are sequences that are part of $B 7 L$ but not present in $B 7 p$

B1 CGGCTCCCATGAGTGTCCTATTCCCAGATGACCCCCGTGAACCCTGCGACGCGTGGCTTG

B2

B3

B4p

B5

B6

B7P

B7L

B1

B2

B3

B4p

B5

B6

B7P

B7L

B1

B2

B3

B4p

B5

B6

B7P

B7L

B1

B2

B3

B4p

B5

B6

B7P

B7L

B1

B2

B3

B4p

B5

B6

B7P

B7L

B1

B2

B3

B4p

B5

B6

B7P

B7L

B1

B2

B3

B4p

B5

B6

B7P

B7L

TGGCTCCCATGAGTGT - CCTATACCTCACGACCCCTGTGAACCCTGTGGCGCCCGGCTTG TGGCTCCCATGAATGT - CCTATCCCTGATGACCCCCGTGAACCCTGCGGCGCCTGGCTTG

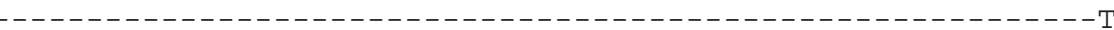
TGGCTCCCATGAGCGTCCTATTCCCAGAAGACCTCCGAGAACCCTGCGGCACCTGGCTTG TGGCTCCCATGAGTGT - TCTATCCCTCACTACCCCCGTGAACCTTGCGGCACCTAGCTTG - . TTAGTCGCATGAGTGT - CTATCTCTGACAACCCTCGGGAACCAATGCGGCACCTGGCCTG

GGTGACCCGGGACGTTTCCACGGGCGAACCCCAGTTGGGGCGGGCTCGGGCTGTGATGTG GATGACCTGGGATGTTTCCACGGGCGAACCCCAGCTGGGGCGGGCTCAGGCTGTGATGTG CGGGACCCGGGACGTTTCCGCGGGCGAACCCCAGCTGGGGCGGGCTCGGGCTGTGATGTG GTTGACCCGGGATGTTTCTGCAGGCAAACC-ACAGGTAGCCGGGCTCGGGCTATGACGCC GGTGACCCAGGACGTTTCCGCGGGCGAACCCCATCCAGGGAGGGCTTGGGCTGTGATGTG CGTGACCCGGGACGTTTCCGCGGGTGAACCTCAGCTGGGGCGGGCTTGAGCTGTGATGTG GGAGACCTGGGACGTTTCTGTGGGTGAACC-CCAGCCAGGCGTGCTCGGGCTGTGATGTG GGAGACCTGGGACGTTTCTGTGGGTGAACC - CCAGCCAGGCGTGCTCGGGCTGTGATGTG

TGAGCCTGCACTGCCGCCGCGCGGCCACTT CGATTCCACAACTACAACCAATTCCGTGC TGAGCCTGCACTGCCGCCGCGCAGCCACCT CGATTCCACAACTACAACCAATTCTGTGC TGAGCCTGCACTGCCGCCACGCGGCCACTTECGATTCCACAGCTACAACCAATTCTGCAC TGAGCCTGCCCTGCTACCACGTGGCCACCT FTGTTTCCATACCTTCAACTGATTCCGTGG TGAGCCTGCACTGCCGCCGCGCGGCCACTT TGAGTCCACACTTACAACCGATTCTGTGC GGAGCCTGCACTGCCGCCGCGCGGCCACTT CGATTCCACAACTACAACCGATTCTGCAC TGAGCCTGCACTGCCGCCGCGCGGCCACTT TTGATTCCACACGTACAGCCGATTCTGCGC TGAGCCTGCACTGCCGCCGCGCGGCCACTTFTGATTCCACACGTACAGCCGATTCTGCGC

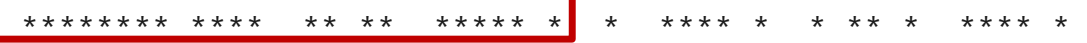

CATCCAGGCGGTGAGACCGAAGGAGAAGCCTCCAGAAATACCCATGTGCTATGCAAGAGC CATCCGGGCGGTGAGACTGAAGGGGAAGCCTCCAGAAATACACATGTGCTATGCAAGAGC CATCCGGGTGGTGAGACTGAAGGGGAAGCCTCCAGAAATATTCATGTGCTATGCAAGAGC

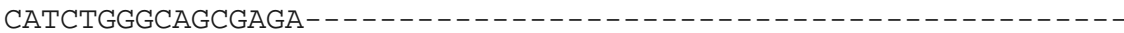
CATCCGGGTGGTGAGACAGAAGGGGAAGCGTCCACAAATACCCATGTGCTACGCAAGAGC CATCGGGGCGGTGAGACCGAAGGGGAAGCCTCCAGAAATACACATGTGCTATGCAAGAGC CATCAGGGCAGTGAGACGGGCCTCCCTTGGTGAGGTCAGCAAGATGGGCCGCCCTCGGTG CATCAGGGCAGTGAGACGGGCCTCCCTTGGTGAGGTCAGCAAGATGGGCCGCCCTCGGTG $* * * * * * \quad * * * * *$

CCCCGGGCT - CTGTGCCTGCCAGAGACCCTGCGAGAAGGGTTTCACACTAGGGCCGAGAT CCCCGGGCT - CTGTGCCTGCCAGAGACCCTGCAAGAAGGATCTCACCCTGAGGCCAAGAT CCCTGGGCTCTTGAGCCTGCCAGAGACCCTGTGGGAGGGGTCTCACGCTGAGGCCGAGAT - - - - - - - - - - - - - - - - - - - - - - - - - - - - - - - - - CCCCAGTTT - CTGTGCCTGCCAGAGACCCTGTGGGAGGGGCCTCACGCTGAGGCCGAGAT CCCCAGGCT - CTGTGCCTGCCAGAGACCCTGTAGGAGGGGTCTCACGCTTAGGCCGAGAT AGGCTGGCATCCAAGACTGAATGGGAAGCCTCCAGAAATACCTGTGCGCTGTGCTT - - - AGGCTGGCATCCAAGACTGAATGGGAAGCCTCCAGAAATACCTGTGCGCTGTGCTTGGCA

CCССАTCATCCATGGAACTGGGACACCTCATTGTTCCСCTAGTATCTCCTCCСАTCTCCC CCCCATCATCCAAAGAATGGGGACACTTCATTGTCCCCCAAGTATCTCСTCTCATCTCCC CCCCATCATCCAAAGAATGGGGACACTTCACTGTCCCCCAAGTATCTCСTCTCATCTCCC - - - - - - - - - - - - - - - - - - - - - - - - - - - - - - - - - - - - - - CCCCATCATCCAAAGAATGGGGACACCTCATTGTCACCAAAGTATCTCATCTC - - - - CC CCСTATCATCCAAGGAACTGGGACACCTCATTGTCCCCCAAGCATCTCCTCCAATCTCCC GGCTCCCACGATCCCTGTTCCTGCTG

CAACCTTCAACAGTGCACCCTGGGGTGAATCAGACACGTAGCCCGATA CAACCCTCAATAGTGCACCCTGGGGTGAATCAGACACATAGCCCGATG CAACCTTCAACAGTGCACCCTGGGGTGAATCAGGCACGTAGCCCGATG - - - - - - - - - - - - - - - - - - - - - - - - - - - - CAACCCTCAATAGTGCACCCTGGGGTGAATCAGACACGTAGCCCAATG CAACCTTCAACAATGCACCCTGGGGTGAATCAAACACATAGCCCGATG - - - -
A rectangle marks the position of sequences that encompass presumed CTCF binding sites. NOTE: according to results of ChIP assays, B2 and B4 do not contain a CTCF site (Fig. 1). In bold are mutations that abolish CTCF-DNA interactions
Shown in yellow is the short deletion within B7L, as detailed in the text; also see a set of alignments shown in Appendix C.
Note Clustal O marks with an * when the nucleotides are identical in the entire set of analyzed sequences 
bioRxiv preprint doi: https://doi.org/10.1101/250407; this version posted January $19,2018$. The copyright holder for this preprint (which was not certified by peer review) is the author/funder, who has granted bioRxiv a license to display the preprint in perpetuity. It is made available under aCC-BY-ND 4.0 International license.

Appendix C: Clustal 0 alignments of B1, B2, B3, B5, B6, B7L

Highlighted in blue are sequences that were added to B7p, producing B7L

B1 CGGCTCCCATGAGTGTCCTATTCCCAGATGACCCCCGTGAACCCTGCGACGCGTGGCTTG

B2

B3

B5

B6

B7L

B1

B2

B3

B5

B6

B7L

B1

B2

B3

B5

B6

B7L

B1

B2

B3

B5

B6

B7L

B1

B2

B3

B5

B6

B7L

B1

B2

B3

B5

B6

B7L

B1

B2

B3

B5

B6

B7L

TGGCTCCCATGAGTGTC-CTATACCTCACGACCCCTGTGAACCCTGTGGCGCCCGGCTTG TGGCTCCCATGAATGTC - CTATCCCTGATGACCCCCGTGAACCCTGCGGCGCCTGGCTTG TGGCTCCCATGAGCGTCCTATTCCCAGAAGACCTCCGAGAACCCTGCGGCACCTGGCTTG TGGCTCCCATGAGTGT - TCTATCCCTCACTACCCCCGTGAACCTTGCGGCACCTAGCTTG TTAGTCGCATGAGTGTC - TATCTCTGACAACCCTCGGGAACCAATGCGGCACCTGGCCTG $* * * * * * * * * * * * * * * * * * * * * * * *$

GGTGACCCGGGACGTTTCCACGGGCGAACCCCAGTTGGGGCGGGCTCGGGCTGTGATGTG GATGACCTGGGATGTTTCCACGGGCGAACCCCAGCTGGGGCGGGCTCAGGCTGTGATGTG CGGGACCCGGGACGTTTCCGCGGGCGAACCCCAGCTGGGGCGGGCTCGGGCTGTGATGTG GGTGACCCAGGACGTTTCCGCGGGCGAACCCCATCCAGGGAGGGCTTGGGCTGTGATGTG CGTGACCCGGGACGTTTCCGCGGGTGAACCTCAGCTGGGGCGGGCTTGAGCTGTGATGTG GGAGACCTGGGACGTTTCTGTGGGTGAACCCCAG-CCAGGCGTGCTCGGGCTGTGATGTG $* * * * * * * * * * *$ ************************t

TGAGCCTGCACTGCCGCCGCGCGGCCACTTCCGATTCCACAACTACAACCAATTCCGTGC TGAGCCTGCACTGCCGCCGCGCAGCCACCTCCGATTCCACAACTACAACCAATTCTGTGC TGAGCCTGCACTGCCGCCACGC $\bar{G} G C C A C \bar{T} T C C G A T T C C A C A G C T A C A A C C A A T T C T G C A C$ TGAGCCTGCACTGCCGCCGCGCGGCCACTTTTGAGTCCACACTTACAACCGATTCTGTGC GGAGCCTGCACTGCCGCCGCGCGGCCACTTCCGATTCCACAACTACAACCGATTCTGCAC TGAGCCTGCACTGCCGCCGCGCGGCCACTTCTGATTCCACACGTACAGCCGATTCTGCGC $* * * * * * * * * * * * * * * * * * * * * * * * * * * * * * * * * * * * * * * * * * * * * *$

CATCCAGGCGGTGAGACCGAAGGAGAAGCCTCCAGAAATACCCATGTGCTATGCAAGAGC CATCCGGGCGGTGAGACTGAAGGGGAAGCCTCCAGAAATACACATGTGCTATGCAAGAGC CATCCGGGTGGTGAGACTGAAGGGGAAGCCTCCAGAAATATTCATGTGCTATGCAAGAGC CATCCGGGTGGTGAGACAGAAGGGGAAGCGTCCACAAATACCCATGTGCTACGCAAGAGC CATCGGGGCGGTGAGACCGAAGGGGAAGCCTCCAGAAATACACATGTGCTATGCAAGAGC CATCAGGGCAGTGAGACGGGCCTCCCTTGGTGAGGTCAGCAAGATGGGCCGCCCTCGGTG $* * * * * * * * * * * * * * * * * * *$

CCCCGGGCT - CTGTGCCTGCCAGAGACCCTGCGAGAAGGGTTTCACACTAGGGCCGAGAT CCCCGGGCT - CTGTGCCTGCCAGAGACCCTGCAAGAAGGATCTCACCCTGAGGCCAAGAT CCCTGGGCTCTTGAGCCTGCCAGAGACCCTGTGGGAGGGGTCTCACGCTGAGGCCGAGAT CCCCAGTTT - CTGTGCCTGCCAGAGACCCTGTGGGAGGGGCCTCACGCTGAGGCCGAGAT CCCCAGGCT - CTGTGCCTGCCAGAGACCCTGTAGGAGGGGTCTCACGCTTAGGCCGAGAT AGGCTGGCATCCAAGACTGAATGGGAAGCCTCCAGAAATACCTGTGCGCTGTGCTTGGCA

CCCCATCATCCATGGAACTGGGACACCTCATTGTTCCCCTAGTATCTCCTCCСATCTCCC CCCCATCATCCAAAGAATGGGGACACTTCATTGTCCCCCAAGTATCTCCTCTCATCTCCC CCCCATCATCCAAAGAATGGGGACACTTCACTGTCCCCCAAGTATCTCСTCTCATCTCCC CCCCATCATCCAAAGAATGGGGACACCTCATTGTCACCAAAGTATCTCATCTC - - - - - CC CССTATCATCCAAGGAACTGGGACACCTCATTGTCCCCCAAGCATCTCСTCCAATCTCCC

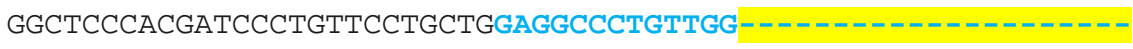

*

CAACCTTCAACAGTGCACCCTGGGGTGAATCAGACACGTAGCCCGATA CAACCCTCAATAGTGCACCCTGGGGTGAATCAGACACATAGCCCGATG CAACCTTCAACAGTGCACCCTGGGGTGAATCAGGCACGTAGCCCGATG CAACCCTCAATAGTGCACCCTGGGGTGAATCAGACACGTAGCCCAATG CAACCTTCAACAATGCACCCTGGGGTGAATCAAACACATAGCCCGATG GAGGGTCTCCCTCTGGACCTTGGGCTCAAGTTCTTTGGGGTCCAAGTC $\star * \star * * \star * * * * * *$
Extent of conservation among sequences that encompass predicted CTCF sites.

B2 does not include a CTCF site. Sequences of CTCF sites in B1, B5, and B7 are identical.
Yellow highlights internal deletions in $\mathrm{B} 5$ and B7L 
bioRxiv preprint doi: https://doi.org/10.1101/250407; this version posted January 19,2018 . The copyright holder for this preprint (which was not certified by peer review) is the author/funder, who has granted bioRxiv a license to display the preprint in perpetuity. It is made available under aCC-BY-ND 4.0 International license.

Appendix D: CLUSTAL 0 alignment of B1, B2, B3, B5, and B6

B1

B2

B3

B5

B6

B1

B2

B3

B5

B6

B1

B2

B3

B5

B6

B1

B2

B3

B5

B6

B1

B2

B3

B5

B6

B1

B2

B3

B5

B6

B1

B2

B3

B5

B6

CGGCTCCCATGAGTGTCCTATTCCCAGATGACCCCCGTGAACCCTGCGACGCGTGGCTTG TGGCTCCC - ATGAGTGTCCTATACCTCACGACCCCTGTGAACCCTGTGGCGCCCGGCTTG TGGCTCCC - ATGAATGTCCTATCCCTGATGACCCCCGTGAACCCTGCGGCGCCTGGCTTG TGGCTCCCATGAGCGTCCTATTCCCAGAAGACCTCCGAGAACCCTGCGGCACCTGGCTTG TGGCTCCC - ATGAGTGTTCTATCCCTCACTACCCCCGTGAACCTTGCGGCACCTAGCTTG

GGTGACCCGGGACGTTTCCACGGGCGAACCCCAGTTGGGGCGGGCTCGGGCTGTGATGTG GATGACCTGGGATGTTTCCACGGGCGAACCCCAGCTGGGGCGGGCTCAGGCTGTGATGTG CGGGACCCGGGACGTTTCCGCGGGCGAACCCCAGCTGGGGCGGGCTCGGGCTGTGATGTG GGTGACCCAGGACGTTTCCGCGGGCGAACCCCATCCAGGGAGGGCTTGGGCTGTGATGTG CGTGACCCGGGACGTTTCCGCGGGTGAACCTCAGCTGGGGCGGGCTTGAGCTGTGATGTG $* * * * * * * * * * * * * * * * * * * * * * * * \quad * * * * * * * * \quad * * * * * * * * * * *$

TGAGCCTGCACTGCCGCCGCGCGGCCACTTCCGATTCCACAACTACAACCAATTCCGTGC TGAGCCTGCACTGCCGCCGCGCAGCCACCTCCGATTCCACAACTACAACCAATTCTGTGC TGAGCCTGCACTGCCGCCACGCGGCCACTTCCGATTCCACAGCTACAACCAATTCTGCAC TGAGCCTGCACTGCCGCCGCGCGGCCACTTTTGAGTCCACACTTACAACCGATTCTGTGC GGAGCCTGCACTGCCGCCGCGCGGCCACTTCCGATTCCACAACTACAACCGATTCTGCAC

CATCCAGGCGGTGAGACCGAAGGAGAAGCCTCCAGAAATACCCATGTGCTATGCAAGAGC CATCCGGGCGGTGAGACTGAAGGGGAAGCCTCCAGAAATACACATGTGCTATGCAAGAGC CATCCGGGTGGTGAGACTGAAGGGGAAGCCTCCAGAAATATTCATGTGCTATGCAAGAGC CATCCGGGTGGTGAGACAGAAGGGGAAGCGTCCACAAATACCCATGTGCTACGCAAGAGC CATCGGGGCGGTGAGACCGAAGGGGAAGCCTCCAGAAATACACATGTGCTATGCAAGAGC $* * * * * * * * * * * * * * * * * * * * * * * * * * * * * * \quad * * * * * * * * * * * * * * * *$

CCCCGGGCTC - TGTGCCTGCCAGAGACCCTGCGAGAAGGGTTTCACACTAGGGCCGAGAT CCCCGGGCTC - TGTGCCTGCCAGAGACCCTGCAAGAAGGATCTCACCCTGAGGCCAAGAT CCCTGGGCTCTTGAGCCTGCCAGAGACCCTGTGGGAGGGGTCTCACGCTGAGGCCGAGAT CCCCAGTTTC - TGTGCCTGCCAGAGACCCTGTGGGAGGGGCCTCACGCTGAGGCCGAGAT CCCCAGGCTC - TGTGCCTGCCAGAGACCCTGTAGGAGGGGTCTCACGCTTAGGCCGAGAT

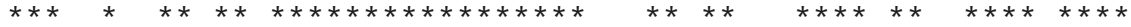

CCCСATCATCCATGGAACTGGGACACCTCATTGTTCCССTAGTATCTCCTCCСATCTCCC CCCCATCATCCAAAGAATGGGGACACTTCATTGTCCCCCAAGTATCTCCTCTCATCTCCC CCCCATCATCCAAAGAATGGGGACACTTCACTGTCCCCCAAGTATCTCCTCTCATCTCCC CCCCATCATCCAAAGAATGGGGACACCTCATTGTCACCAAAGTATCTCATC - - - - - TCCC CCCTATCATCCAAGGAACTGGGACACCTCATTGTCCCCCAAGCATCTCCTCCAATCTCCC

CAACCTTCAACAGTGCACCCTGGGGTGAATCAGACACGTAGCCCGATA CAACCCTCAATAGTGCACCCTGGGGTGAATCAGACACATAGCCCGATG CAACCTTCAACAGTGCACCCTGGGGTGAATCAGGCACGTAGCCCGATG CAACCCTCAATAGTGCACCCTGGGGTGAATCAGACACGTAGCCCAATG CAACCTTCAACAATGCACCCTGGGGTGAATCAAACACATAGCCCGATG $* * * * * * * * * * * * * * * * * * * * * * * * * * * * * \quad * * * * * * * * * * *$ 